\title{
Associations between arterial stiffness and blood pressure fluctuations after spinal cord injury
}

\author{
Katharine D. Currie ${ }^{1,2} \cdot$ Michèle Hubli $^{2,3} \cdot$ Maureen J. MacDonald ${ }^{4}$ Andrei V. Krassioukov ${ }^{2,5,6}$
}

Received: 7 February 2019 / Revised: 24 April 2019 / Accepted: 2 May 2019 / Published online: 19 June 2019

(c) The Author(s), under exclusive licence to International Spinal Cord Society 2019

\begin{abstract}
Study design Cross-sectional.

Objective To examine the relationship between arterial stiffness and daily fluctuations in blood pressure (BP) owing to hypotensive events and autonomic dysreflexia (AD) in individuals with a T6 and above spinal cord injury (SCI).

Setting University-based laboratory in Vancouver, BC, Canada.

Methods Twenty-six individuals (73\% male; 43 (11) years) with a chronic (>1 year post SCI), traumatic, motor-complete SCI with a neurological level of injury of C4-T6 participated in this study. Arterial stiffness was assessed using carotid-tofemoral pulse wave velocity (cfPWV). BP was measured over a 24-hr period using ambulatory BP monitoring. AD was defined as an increase in systolic BP $>20 \mathrm{mmHg}$ above baseline BP. Hypotensive events were defined as a decrease in systolic $\mathrm{BP} \geq 20 \mathrm{mmHg}$ and/or diastolic $\mathrm{BP} \geq 10 \mathrm{mmHg}$ below baseline. The severity and frequency of these events were quantified and Pearson and Spearman's correlations between them and cfPWV were performed.

Results $\mathrm{AD}$ severity and frequency were not were correlated with cfPWV. For hypotensive events, both the frequency $(r=0.412, P=0.04)$ and severity ( $\Delta$ systolic BP; $r=-0.425, P=0.03)$ of these events were correlated with cfPWV. The combined total of $\mathrm{AD}$ and hypotensive events (9 (5) events/day) was also correlated with cfPWV $(r=0.480, P=0.01)$.

Conclusions Hypotensive events, and the combined frequency of both hypo- and hypertensive events within a 24-hr period are associated with increased arterial stiffness in individuals with T6 and above SCI, suggesting BP instability may play a role in arterial stiffening post SCI.
\end{abstract}

Andrei V. Krassioukov

krassioukov@icord.org

1 Department of Kinesiology, Michigan State University, East Lansing, MI, USA

2 International Collaboration on Repair Discoveries (ICORD), Department of Medicine, University of British Columbia, Vancouver, BC, Canada

3 Spinal Cord Injury Center, Balgrist University Hospital, University of Zurich, Zurich, Switzerland

4 Department of Kinesiology, McMaster University, Hamilton, ON, Canada

5 Division of Physical Medicine and Rehabilitation, Department of Medicine, University of British Columbia, Vancouver, BC, Canada

6 G. F. Strong Rehabilitation Centre, Vancouver, BC, Canada

\section{Introduction}

Motor-complete spinal cord injury (SCI) at T6 and above are typically associated with extreme daily fluctuations in blood pressure (BP), which are attributed to the loss of supraspinal control over spinal sympathetic outflow to the vasculature [1]. These fluctuations include both severe increases in BP attributed to autonomic dysreflexia (AD), and decreases in BP (i.e., hypotensive events) commonly resulting from postural changes. Although hypotensive events occur in the uninjured population (i.e., individuals without a $\mathrm{SCI}$ ), $\mathrm{AD}$ is unique to $\mathrm{SCI}$ and describes episodic hypertension elicited by noxious or non-noxious stimuli below the level of injury [1].

In the uninjured population, hypertension [2] and postural hypotension [3] are both associated with increased carotid-to-femoral pulse wave velocity (cfPWV), which is the gold-standard measurement of arterial stiffness [4]. Miyatani et al. [5] demonstrated those with SCI who have higher resting BP have higher cfPWV than their SCI peers 
with normal BP. Recently, a comparison of arterial stiffness between individuals with SCI who self-reported postural hypotension or $\mathrm{AD}$ observed increased arterial stiffness in the ones reporting postural hypotension [6]. This study did not measure the severity and frequency of these hypotensive and $\mathrm{AD}$ events. Therefore, the purpose of this study was to examine the relationship between cfPWV and daily BP fluctuations through the quantitative assessment of both hypertensive (i.e., AD) and hypotensive events over a $24-\mathrm{hr}$ period. We hypothesized that individuals who experienced more BP instability would have increased cfPWV.

\section{Methods}

\section{Participants}

Eligible individuals for this study were recruited from a larger study examining the effects of two different exercise interventions on cardiovascular health [7], and all measurements were collected prior to the initiation of exercise training. Inclusion criteria included males and females that were $>18$ years of age who had a traumatic, chronic $(\geq 1$ year post injury), motor-complete [American Spinal Cord Injury Impairment Scale (AIS) A or B] SCI with a neurological level of injury (NLI) of C4-T6, which were determined using the International Standards for Neurological Classification of SCI [8]. Exclusion criteria included any self-reported history and/or symptoms of cardiovascular or cardiopulmonary disease, which was identified using a questionnaire, major trauma or surgery within 6 months, fracture within 12 months, active stage 3 or 4 pressure ulcers, any unstable medical/psychiatric condition or substance abuse disorder, and any cognitive dysfunction or language barrier that prevented them from following English instructions. All procedures conformed to the Declaration of Helsinki and were approved by the University of British Columbia's Clinical Research Ethics Board (H12-02910), and all participants provided written informed consent.

\section{Study protocol}

This was a cross-sectional study design that was conducted in the Autonomic Research Laboratory in the International Collaboration on Repair Discoveries (Vancouver, BC, Canada). Participants were recruited from the community between 2014 and 2017 and attended one laboratory visit for the assessment of cfPWV. After this visit, they were set up with a 24-hr ambulatory blood pressure monitoring (ABPM) device. Prior to their laboratory visit, participants were instructed to abstain from food and drink for $4 \mathrm{~h}$, caffeine and alcohol for $12 \mathrm{~h}$, and exercise for $24 \mathrm{~h}$ prior to testing. Individuals were also asked to void their bladders prior to the measurement to reduce the influence of sympathetic reflex activation on BP. At total of 35 individuals were tested, but eight were excluded due to incomplete 24-ABPM measurements and one was excluded owing to the use of alpha-1-agonist, which may have had an effect on his BP responses.

\section{cfPWV assessment}

cfPWV was measured as previously described [9]. In brief, following 10 mins of supine rest, a minimum of 30-s of carotid and femoral arterial pressure waveforms were collected simultaneously from the right side of the body using hand-held tonometers (Model SPT-301; Millar Instruments Inc., Houston, TX). Arterial pressure waveforms were bandpass filtered $(2-30 \mathrm{~Hz})$, and the arrival of the waveform at each site was identified as the minimum value of the filtered signal. A 10-s sample of waveforms was analyzed, and the difference between the arrival of the waveform at each site was calculated as the pulse transit time $(\Delta \mathrm{t})$. The direct distance $(D)$ between the carotid and femoral sites was measured along the surface of the body using anthropometric tape. Real cfPWV was calculated as $(0.8 \times D) / \Delta \mathrm{t}$, and is reported as the average from two, 10-s waveform sections. If the difference between the cfPWV values was $>0.5 \mathrm{~m} \mathrm{~s}^{-1}$, a third 10 -s section was analyzed and the median cfPWV value is reported. We have previously demonstrated this measurement to have almost perfect interand intra-rater reliability in individuals with SCI [9].

\section{4-hr ABPM assessment}

BP was measured across a 24-hr period using an ABPM device (Meditech Card (X) plore; Meditech Ltd., Budapest, Hungary) as previously described [10]. Automatic BP recordings were taken from the non-dominant arm every $15 \mathrm{~min}$ from 0800 to $1900 \mathrm{~h}$ (daytime period), and every $60 \mathrm{~min}$ from 1900 to $0800 \mathrm{~h}$ (nighttime period). Participants were instructed to initiate a manual measurement during any activity that may trigger $\mathrm{AD} /$ hypotension, or anytime they experienced symptoms of $\mathrm{AD} / \mathrm{hypotension}$. These measurements, along with other events (i.e., awake and sleep time, eating, exercise, etc.) were documented in an activity $\log$

Baseline daytime systolic BP (SBP) and diastolic BP (DBP) were calculated by averaging three successive automatic measurements after the participant was awake and had transferred to their chair for the day. These measurements took place between 0900 and $1430 \mathrm{~h}$. These values were subsequently used as a reference point to assess the frequency and severity of $\mathrm{AD}$ and hypotensive events during the daytime. AD was defined as an increase in SBP 
$>20 \mathrm{mmHg}$ above baseline daytime SBP, whereas a hypotensive event was defined as a decrease in SBP $\geq 20$ $\mathrm{mmHg}$ and/or DBP $\geq 10 \mathrm{mmHg}$ below their baseline daytime BPs [10]. Hypotensive events were not calculated during the nighttime because of the potential for a nocturnal dip in BP. AD events during the nighttime were determined using a baseline nighttime SBP, which was calculated as the average of the first three automatic BP measurements when the participant was asleep. Successive BP measurements (either autonomic or manual) that met the criteria for either $\mathrm{AD}$ or hypotensive events were counted as a single event. The frequency of $\mathrm{AD}$ and hypotensive events was calculated as the total number of each event, whereas total BP events was calculated as the sum of all events. The maximum increase in SBP and decrease in SBP and DBP were used to characterize the severity of $\mathrm{AD}$ and hypotensive events, respectively. For AD and hypotensive events that involved successive measurements, the time difference between the first and last measurement was calculated and the longest duration was reported for both $\mathrm{AD}$ and hypotensive events. Finally, the range in SBP and DBP across the 24-hr period was calculated as the difference between the maximum and minimum daily $\mathrm{BP}$ values.

\section{Statistical analysis}

Statistical analyses were performed using Statistical Package for the Social Sciences (Version 20.0; IBM Corporation, Armonk, NY, USA). Data were assessed for normality using Shapiro-Wilk tests, and analyzed using Pearson or Spearman's correlations for normal and non-normal distributed data, respectively. All statistical analyses were considered significant at $P<0.05$.

\section{Results}

Twenty-six individuals participated in the study. Participant characteristics are described in Table 1 . The majority of participants were male $(73 \%)$ and had tetraplegia $(58 \%)$. The average cfPWV was $7.9(1.5) \mathrm{m} / \mathrm{s}$ (range 4.6-11.7). Abnormal cfPWV values (i.e., higher than reference sample) were present in $81 \%$ of the sample when using real cfPWV uninjured reference values [11] and 58\% of the sample when using real cfPWV SCI reference values [5]. These reference values compared with the values from our sample are presented in Fig. 1.

A summary of the 24-hr ABPM data including AD and hypotensive events, and their correlations with cfPWV is presented in Table 2. The relationships between BP event frequency and cfPWV is also illustrated in Fig. 2. No AD parameters were independently related to cfPWV. The severity of hypotensive events, quantified as the maximum
Table 1 Participant characteristics

\begin{tabular}{llll}
\hline Variable & $\begin{array}{l}\text { Total } \\
(n=26)\end{array}$ & $\begin{array}{l}\text { Tetraplegia } \\
(n=15)\end{array}$ & $\begin{array}{l}\text { Paraplegia } \\
(n=11)\end{array}$ \\
\hline Age (years) & $43(11)$ & $44(12)$ & $42(11)$ \\
Sex: male, female $(n)$ & 19,7 & 10,5 & 9,2 \\
Weight $(\mathrm{kg})$ & $72.8(16.2)$ & $72.7(16.1)$ & $73.2(17.2)$ \\
Height $(\mathrm{m})$ & $1.77(0.12)$ & $1.76(0.09)$ & $1.72(0.07)$ \\
Lesion level & $\mathrm{C} 4-\mathrm{T} 5$ & $\mathrm{C} 4-\mathrm{C} 6$ & $\mathrm{~T} 2-\mathrm{T} 5$ \\
AIS: A, B $(n)$ & 18,8 & 7,8 & 11,0 \\
$\begin{array}{l}\text { Time post injury } \\
\text { (years) }\end{array}$ & $15(11)$ & $15(10)$ & $14(14)$ \\
Medication drug class & $(n)$ & & \\
Beta-3-adrenergic & 1 & 1 & 0 \\
agonist & & 1 & 2 \\
Analgesics & 3 & 1 & 0 \\
$\begin{array}{l}\text { Anti-convulsant } \\
\text { Anti-depressant }\end{array}$ & 1 & 2 & 1 \\
Anti-spasmodic & 2 & 1 & 1 \\
Gamma- & 9 & & \\
aminobutyric & & & \\
acid analog & & & \\
\hline
\end{tabular}

Variables are presented as mean (SD). A motor- and sensory-complete, AIS American Spinal Cord Injury Impairment Scale, $B$ motor-complete and sensory-incomplete, $C$ cervical, $T$ thoracic

decrease in SBP was negatively correlated with cfPWV $(r=-0.425, P=0.030)$, whereas the frequency of hypotensive events was positively correlated $(r=0.412, P=$ 0.036). cfPWV was also positively correlated with the total number of BP events $(r=0.480, P=0.013)$. The activities reported during hypotensive events including transfers and changes in body position (e.g., waking up in the morning), eating and removing an abdominal binder. The activities reported during $\mathrm{AD}$ events included transfers, catheterization and voiding bladder, bowel movements and muscle spasms.

\section{Discussion}

Our preliminary findings demonstrate a link between the severity and frequency of hypotensive events and arterial stiffening in individuals with T6 and above SCI. Furthermore, those who experience more BP instability, as indicated by the total number of hypotensive and $\mathrm{AD}$ events, also have increased arterial stiffness. However, no significant relationship was observed between cfPWV and AD within a 24-hr period. The evidence for the role of cfPWV as an indicator of cardiovascular health in individuals with SCI has been steadily growing owing to the demonstrated clinical significance of this assessment. cfPWV is an independent predictor of cardiovascular morbidity and mortality 


\section{a}

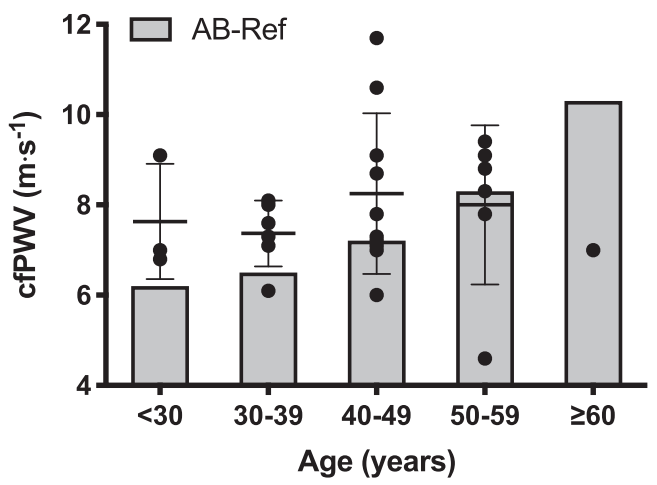

Fig. 1 Comparison of individual carotid-to-femoral pulse wave velocity (cfPWV) values to published reference values. a Reference values for uninjured individuals (AB-Ref) separated by decade of age are presented as the gray bar [11]. Black circles represent the participants from this study with the mean (SD) when applicable. b Reference b

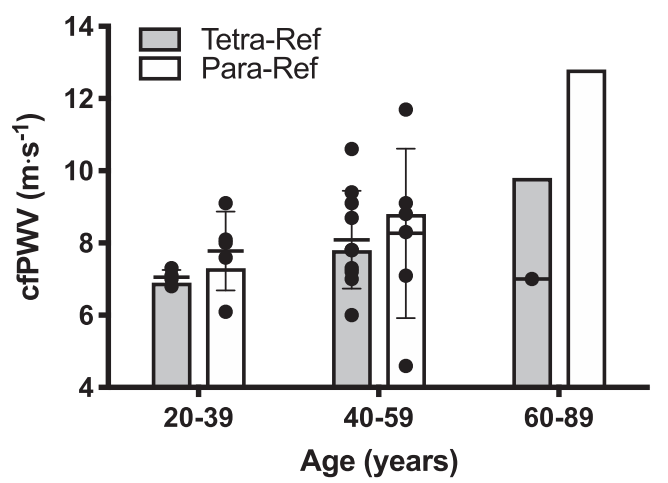

values for individuals with tetraplegia (Tetra-Ref) and paraplegia (Para-Ref) represented as gray and white boxes, respectively for three age categories [5]. Black circles represent participants from this study separated based on lesion level and age, with mean (SD) when applicable
Table 2 24-hr ambulatory blood pressure monitoring values and their relationship to carotid-tofemoral pulse wave velocity

\begin{tabular}{lllll}
\hline Variable & Mean (SD) & Range & Correlation coefficient & $P$ value \\
\hline AD events & & & & \\
Frequency (events/day) & $5(3)$ & $0-13$ & 0.219 & 0.28 \\
Maximum SBP (mmHg)* & $154(27)$ & $110-226$ & 0.177 & 0.39 \\
Maximum $\Delta$ SBP (mmHg)* & $49(27)$ & $14-119$ & 0.041 & 0.84 \\
Longest AD period (min)* & $25(23)$ & $0-88$ & 0.036 & 0.86 \\
Hypotensive events & & & & \\
Frequency (events/day) & $5(4)$ & $0-14$ & 0.412 & $\mathbf{0 . 0 4}$ \\
Minimum SBP (mmHg) & $82(9)$ & $64-94$ & -0.069 & 0.74 \\
Minimum DBP (mmHg) & $44(8)$ & $27-57$ & 0.358 & 0.07 \\
Maximum $\Delta$ SBP (mmHg) & $-23(8)$ & $-37--11$ & -0.425 & $\mathbf{0 . 0 3}$ \\
Maximum $\Delta$ DBP (mmHg) & $-19(8)$ & $-38--7$ & -0.128 & 0.53 \\
Longest hypotensive period (min)* & $29(34)$ & $0-135$ & 0.211 & 0.30 \\
Total BP events (events/day) & $9(5)$ & $3-21$ & 0.480 & $\mathbf{0 . 0 1}$ \\
Daily SBP range (mmHg) & $76(28)$ & $35-149$ & 0.262 & 0.20 \\
Daily DBP range (mmHg)* & $57(20)$ & $30-101$ & 0.186 & 0.36 \\
\hline
\end{tabular}

$A D$ autonomic dysreflexia, $B P$ blood pressure, $D B P$ diastolic blood pressure, $S B P$ systolic blood pressure *Indicates variable was analyzed using Spearman's correlation. All other variables were analyzed using Pearson's correlation. Bold $P$ values indicate significant correlation in the uninjured community [4], and as cardiovascular disease is the leading cause of mortality in the SCI community [12], cfPWV may serve as an important prognostic tool. Studies to date have consistently demonstrated individuals with SCI have increased cfPWV relative to their uninjured peers [5, 13-15], and although most assess resting $\mathrm{BP}$, none have examined the impact of daily BP fluctuations on arterial stiffness. Our sample demonstrated cfPWV values higher than reference values in $81 \%$ of the sample compared with uninjured individuals and $58 \%$ of the sample compared with those with SCI. The comparison to SCI reference values, however, should be interpreted with caution given the reference sample included injury levels below $\mathrm{T} 6$ and both motor-complete and motor-incomplete injuries. Therefore, this prevalence may be slightly inflated. Nonetheless, the comparison with uninjured reference values provides further support that individuals with SCI have elevated arterial stiffness relative to uninjured individuals of a similar age and suggest that daily BP events which are unique to SCI may be a contributing factor.

Lower BP (i.e., postural hypotension) is hypothesized to be caused by arterial stiffness and the resultant reduction in baroreflex sensitivity [3]. Evidence in support of this is provided by Phillips et al. [16] who observed postural 

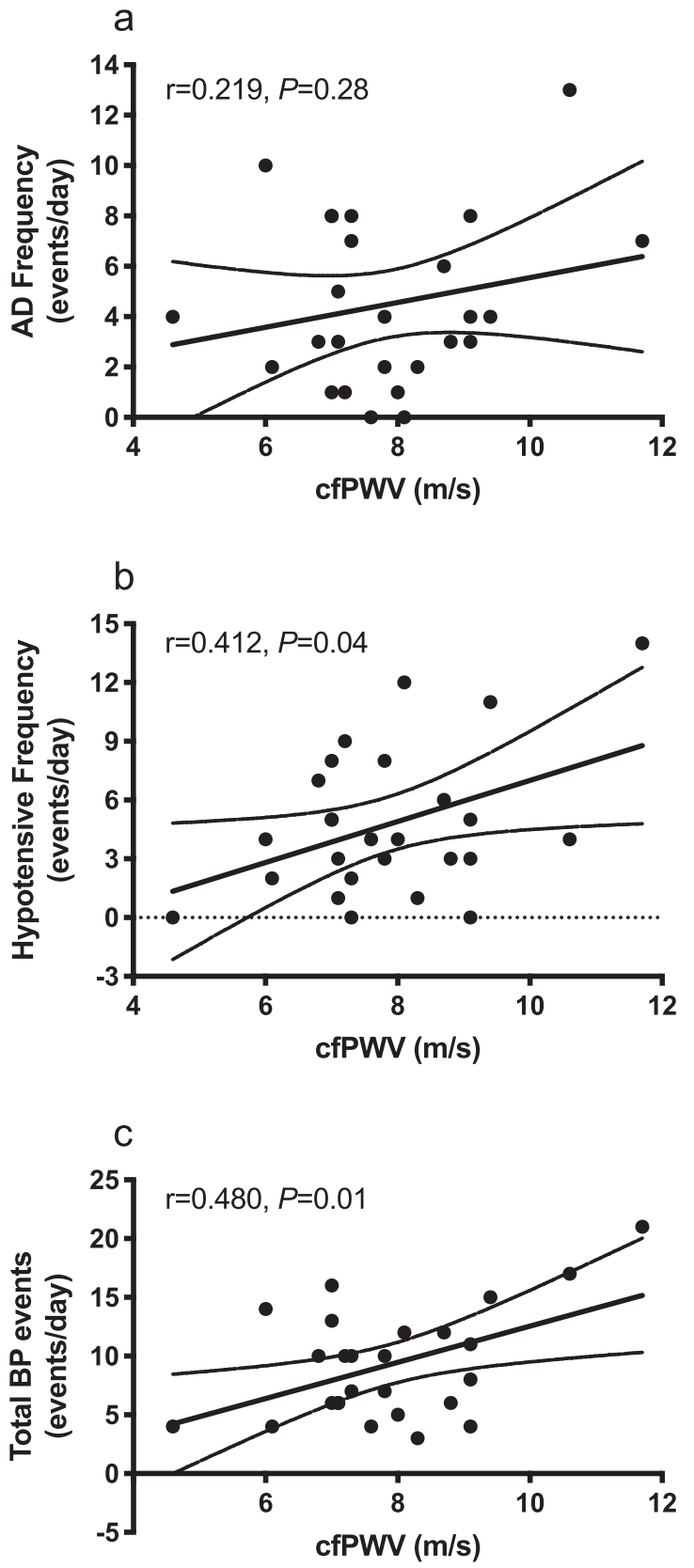

Fig. 2 Correlations between carotid-to-femoral pulse wave velocity (cfPWV) and frequency of blood pressure events across a 24-hr period including a autonomic dysreflexia (AD), b hypotensive events, and $\mathbf{c}$ the total number of $\mathrm{AD}$ and hypotensive events

hypotension, increased carotid artery $\beta$-stiffness and decreased baroreflex sensitivity in individuals with cervical SCI compared with an uninjured control group. Central arterial stiffness measured using aortic augmentation index has been shown to be higher in individuals with SCI who self-reported postural hypotension compared with their peers with SCI who did not report postural hypotension [6]. Our quantitative evaluation of BP events supports this finding as we demonstrate individuals with higher cfPWV experienced more severe and more frequent hypotensive events than those with lower cfPWV. Although these collective observations lend further support that arterial stiffening may contribute to hypotensive events following SCI, it is important to recognize that the primary contributor to hypotension following SCI is likely the interruption of spinal sympathetic outflow to the vasculature [1]. Sympathetic input to the blood vessels in the lower body, including the major vascular beds in the splanchnic region, arise from the sixth thoracic to second lumbar spinal level [17]; therefore SCI above these levels are often associated with cardiovascular dysfunctions including hypotension and AD.

Unlike hypotension, the cause-and-effect relationship between hypertension and arterial stiffening is presently debated. It is known that high BP elicits vascular remodeling, which in turn can contribute to arterial stiffening [18]. The high BP reached during an AD episode is typically transient and should decrease once the stimulus is removed; however, it is the repeated exposure to these hypertensive bouts over time that may provide a substantial stimulus for vascular remodeling. In the present study, we observed no relationship between $\mathrm{AD}$ frequency or severity and cfPWV, which is supported by Katzelnick et al. [6] who observed no relationship between self-reported $\mathrm{AD}$ and aortic augmentation index. Although these findings suggest episodic hypertension owing to AD may not have detrimental effects on cardiovascular health, evidence from animal studies suggests the contrary. In particular, research from our group has demonstrated rodents with SCI that are exposed to six, 10-minute $\mathrm{AD}$ bouts per day experienced impairments in cardiac [19] and cerebrovascular [20] function. In our sample, nine participants $(35 \%)$ had six or more AD episodes per day. Therefore, a larger sample with a greater range of $\mathrm{AD}$ frequency may be necessary to draw more definitive conclusions on the relationship between arterial stiffness and AD. Furthermore, our decision to include both hypotensive and $\mathrm{AD}$ events was to enable examinations of the influence of BP fluctuations on arterial stiffness as we hypothesized that lower BP followed by a higher $\mathrm{BP}$ episode owing to AD may provide a more potent stimulus for vascular remodeling. While the SBP and DBP ranges in the current study were not related to arterial stiffness, the total number of BP events (i.e., hypotensive and $\mathrm{AD}$ ) in a 24-hr period was positively correlated with cfPWV. These preliminary findings warrant further examination on the combined effects of hypotensive and hypertensive effects on arterial health following SCI.

\section{Limitations}

This was a pilot study examining the possible associations between daily BP fluctuations and arterial stiffness in a sample of individuals with a SCI with a NLI of C4-T6. We 
did not control for physical activity levels [21], which can influence cfPWV, and did not include non-traumatic SCI or those with a NLI of C1-C3. Our sample also had a wide range of time post injury (1-42 years), although we did not observe any relationship between cfPWV and time postinjury (rho $=0.047, P=0.82$ ). Although our heterogenous sample is representative of community members living with a T6 and above SCI, additional larger studies with wider inclusion criteria including non-traumatic SCI and a NLI of C1-C3 are needed to draw more definitive conclusions. Furthermore, these studies should consider the possible influences of physical activity levels and time post injury. We defined any decline in SBP $\geq 20 \mathrm{mmHg}$ or DBP $\geq 10 \mathrm{mmHg}$ as a hypotensive event, rather than postural hypotension, as the cause of the event in individuals with SCI is not always attributed to a postural change. Requiring individuals to trigger a manual BP measurement during a suspected $\mathrm{AD}$ or hypotensive event may have resulted in missed measurements, especially if the BP event was asymptomatic. Continuous BP measurements or 24-ABPM measurements across multiple days would provide a more accurate assessment of the frequency of BP events.

\section{Conclusions}

In conclusion, daily BP fluctuations that are unique to individuals with tetraplegia and high-paraplegia are associated with arterial stiffening. Our preliminary findings demonstrated a positive correlation with the severity and frequency of hypotensive events and the total number of hypotensive and $\mathrm{AD}$ events within a $24-\mathrm{hr}$ period. Furthermore, when compared with reference values, our sample showed increased cfPWV, providing further support that individuals with T6 and above SCI are prone to arterial stiffening. Further research is warranted in order to adequately understand the role of BP fluctuations in arterial stiffening following SCI so that appropriate measures can be taken to mitigate its potential effects.

\section{Data availability}

The data sets generated and/or analyzed during the current study are available from the corresponding author on reasonable request.

Acknowledgements We thank Cameron Gee and Marko Gavric for their assistance with data collection and Chris Shamatutu for his assistance with data management.

Funding This work was supported by a Canadian Institutes of Health Research team grant to A.V. Krassioukov (TCA-118348). K.D. Currie was funded by a fellowship from the Craig H. Neilsen Foundation
(281863), whereas M. Hubli was funded by a fellowship from the Swiss National Science Foundation for Prospective Researchers (P300P3_151153).

Author contributions K.D.C. was responsible for designing the study, preparing the ethics, collecting and analyzing the data, interpreting the results, drafting the manuscript and figures, and approving the final manuscript. M.H. was responsible for designing the study, preparing the ethics, collecting and analyzing the data, interpreting the results, revising and approving the final manuscript. M.J.M. was responsible for designing the study, interpreting the results, revising and approving the final manuscript. A.V.K. was responsible for designing the study, preparing the ethics, recruiting participants, interpreting the results, revising and approving the final manuscript.

\section{Compliance with ethical standards}

Conflict of interests The authors declare that they have no conflict of interest.

Statement of ethics We certify that all applicable institutional and governmental regulations concerning the ethical use of human volunteers were followed during the course of this research.

Publisher's note: Springer Nature remains neutral with regard to jurisdictional claims in published maps and institutional affiliations.

\section{References}

1. Teasell RW, Arnold JM, Krassioukov A, Delaney GA. Cardiovascular consequences of loss of supraspinal control of the sympathetic nervous system after spinal cord injury. Arch Phys Med Rehabil. 2000;81:506-16.

2. Najjar SS, Scuteri A, Shetty V, Wright JG, Muller DC, Fleg JL, et al. Pulse wave velocity is an independent predictor of the longitudinal increase in systolic blood pressure and of incident hypertension in the Baltimore Longitudinal Study of Aging. J Am Coll Cardiol. 2008;51:1377-83.

3. Sung SH, Chen ZY, Tseng TW, Lu DY, Yu WC, Cheng HM, et al. Wave reflections, arterial stiffness, and orthostatic hypotension. Am J Hypertens. 2014;27:1446-55.

4. Vlachopoulos C, Aznaouridis K, Stefanadis C. Prediction of cardiovascular events and all-cause mortality with arterial stiffness: a systematic review and meta-analysis. J Am Coll Cardiol. 2010;55:1318-27.

5. Miyatani M, Szeto M, Moore C, Oh PI, McGillivray CF, Catharine Craven B. Exploring the associations between arterial stiffness and spinal cord impairment: A cross-sectional study. J Spinal Cord Med. 2014;37:556-64.

6. Katzelnick CG, Weir JP, Chiaravalloti ND, Wylie GR, DysonHudson TA, Bauman WA, et al. Impact of blood pressure, lesion level, and physical activity on aortic augmentation index in persons with spinal cord injury. J Neurotrauma. 2017;34:3407-15.

7. Krassioukov AV, Currie KD, Hubli M, Nightingale TE, Alrashidi AA, Ramer L, et al. Effects of exercise interventions on cardiovascular health in individuals with chronic, motor complete spinal cord injury: protocol for a randomised controlled trial [Cardiovascular Health/Outcomes: Improvements Created by Exercise and education in SCI (CHOICES) Study]. BMJ Open. 2019;9: e023540.

8. Kirshblum SC, Burns SP, Biering-Sorensen F, Donovan W, Graves DE, Jha A, et al. International standards for neurological 
classification of spinal cord injury (revised 2011). J Spinal Cord Med. 2011;34:535-46.

9. Currie KD, Hubli M, Krassioukov AV. Applanation tonometry: a reliable technique to assess aortic pulse wave velocity in spinal cord injury. Spinal Cord. 2014;52:272-5.

10. Hubli M, Gee CM, Krassioukov AV. Refined assessment of blood pressure instability after spinal cord injury. Am J Hypertens. 2015;28:173-81.

11. Reference Values for Arterial Stiffness Collaboration. Determinants of pulse wave velocity in healthy people and in the presence of cardiovascular risk factors: "establishing normal and reference values'. Eur Heart J. 2010;31:2338-50.

12. Garshick E, Kelley A, Cohen SA, Garrison A, Tun CG, Gagnon $\mathrm{D}$, et al. A prospective assessment of mortality in chronic spinal cord injury. Spinal Cord. 2005;43:408-16.

13. Phillips AA, Cote AT, Bredin SS, Krassioukov AV, Warburton DE. Aortic stiffness increased in spinal cord injury when matched for physical activity. Med Sci Sports Exerc. 2012; 44:2065-70.

14. Miyatani M, Masani K, Oh PI, Miyachi M, Popovic MR, Craven BC. Pulse wave velocity for assessment of arterial stiffness among people with spinal cord injury: a pilot study. J Spinal Cord Med. 2009;32:72-78.
15. Lee AH, Phillips AA, Krassioukov AV. Increased central arterial stiffness after spinal cord injury: contributing factors, implications, and possible interventions. J Neurotrauma. 2017; 34:1129-40.

16. Phillips AA, Krassioukov AV, Ainslie PN, Cote AT, Warburton DE. Increased central arterial stiffness explains baroreflex dysfunction in spinal cord injury. $\mathrm{J}$ Neurotrauma. 2014;31:1122-8.

17. Krassioukov A. Autonomic function following cervical spinal cord injury. Respir Physiol Neurobiol. 2009;169:157-64.

18. Mitchell GF. Arterial stiffness and hypertension: chicken or egg? Hypertension. 2014;64:210-4.

19. West CR, Squair JW, McCracken L, Currie KD, Somvanshi R, Yuen V, et al. Cardiac consequences of autonomic dysreflexia in spinal cord injury. Hypertension. 2016;68:1281-9.

20. Phillips AA, Matin N, Jia M, Squair JW, Monga A, Zheng MMZ, et al. Transient hypertension after spinal cord injury leads to cerebrovascular endothelial dysfunction and fibrosis. J Neurotrauma. 2018;35:573-81.

21. Hubli M, Currie KD, West CR, Gee CM, Krassioukov AV. Physical exercise improves arterial stiffness after spinal cord injury. J Spinal Cord Med. 2014;37:782-5. 